\title{
GW23-e1205 THERAPEUTIC EFFECTIVENESS AND SAFETY OF IMPLANTED STENTS IN CORONARY HEART DISEASE PATIENTS OVER 70 YEARS OLD
}

doi:10.1136/heartjnl-2012-302920j.50

Wang Yue-Xi, Ren Bao-jun. The Department of Cardiology, First Hospital Affiliated to Inner Mongolian Medical College, Huhhot 010059 China

Objectives Stenting of coronary lesions in patients over 70 years old remains a challenging subset. The purpose of this study was to analyse the therapeutic effectiveness and safety of coronary stenting in patients with coronary heart disease over 70 years old.

Methods Between May 2001 to October 2007, data were collected retrospectively on 416 patients with coronary heart disease treated with coronary stenting in our institution. At-least 6 months follow-up data were obtained and coronary angiogram was performed in the presence of clinical or stress test ischaemia. The database was analysed to exact univariate predictors of 6 months adverse events.

Results A total of 416 patients were included, of 95 patients were over 70 years old (the elder group), of 321 patients were under 70 years old (the younger group). (1) The constituting ratio of pathological change contingents and involved positions among the two groups was not notable difference, $p>0.05$. (2) The ratio of restenosis in stents among the two groups wasn't notable difference, $p>0.05$. (3) The major adverse cardiovascular events (MACE) rate but acute myocardial infarction among the two groups was notable difference over 6 months follow-up, $p<0.01$.

Conclusions The Therapeutic effectiveness and safety of implanted stents in coronary heart disease patients over 70 years old was similar to the younger group, but the MACE was higher than the younger group, but the acute myocardial infarction over at-least 6-month follow-up. 